\title{
Effects of ferulic acid on diabetic nephropathy in a rat model of type 2 diabetes
}

\author{
Ran Choi ${ }^{1}$, Bo Hwan Kim², Jarinyaporn Naowaboot ${ }^{1}$, \\ Mi Young Lee ${ }^{1}$, Mi Ri Hyun ${ }^{3}$, Eun Ju Cho', \\ Eun Soo Lee ${ }^{1}$, Eun Young Lee ${ }^{3}$, \\ Young Chul Yang ${ }^{4}$ and Choon Hee Chung ${ }^{1,5,6}$ \\ ${ }^{1}$ Department of Internal Medicine \\ Yonsei University Wonju College of Medicine \\ Wonju 220-701, Korea \\ ${ }^{2}$ Department of Nursing \\ Gachon University of Medicine and Science \\ Incheon 406-799, Korea \\ ${ }^{3}$ Department of Internal Medicine \\ Soonchunhyang University College of Medicine \\ Cheonan 331-090, Korea \\ ${ }^{4}$ Department of Anatomy \\ ${ }^{5}$ Institute of Lifestyle Medicine \\ Yonsei University Wonju College of Medicine \\ Wonju 220-701, Korea \\ ${ }^{6}$ Corresponding author: Tel, 82-33-741-0506; \\ Fax, 82-33-731-5884; E-mail, cchung @yonsei.ac.kr \\ http://dx.doi.org/10.3858/emm.2011.43.12.078
}

Accepted 23 September 2011

Available Online 6 October 2011

Abbreviations: ACR, albumin-creatinine ratio; AGE, advanced glycation end product; FA, ferulic acid; GBM, glomerular basement membrane; GMI, glomerular matrix index; HOMA-IR, homeostatic model assessment insulin resistance; IPGTT, intraperitoneal glucose tolerance test; IVITT, intravenous insulin tolerance test; LETO, Long-Evans Tokushima Otsuka; MCP-1, monocyte chemoattractant protein-1; MDA, malondialdehyde; OLETF, Otsuka Long-Evans Tokushima Fatty

\begin{abstract}
Diabetic nephropathy is the most serious complication in diabetes mellitus. It is known that oxidative stress and inflammation play a central role in the development of diabetic nephropathy. In this study, we investigated that ferulic acid (FA) known as anti-oxidative agent could effect on diabetic nephropathy by anti-oxidative and anti-inflammatory mechanism. We examined the effects of FA in obese diabetic Otsuka Long-Evans Tokushima Fatty (OLETF) rats and non-diabetic control Long-Evans Tokushima Otsuka (LETO) rats. We treated FA to experimental rats from 26 to 45 weeks of age. We evaluated ACR, MDA and MCP-1 in $24 \mathrm{~h}$ urine
\end{abstract}

and examined renal histopathology and morphologic change in extracted kidneys from rats. Also, we evaluated the ROS production and MCP-1 levels in cultured podocyte after FA treatment. In the FA-treated OLETF rats, blood glucose was significantly decreased and serum adiponectin levels were increased. Urinary ACR was significantly reduced in FA-treated OLETF rats compared with diabetic OLETF rats. In renal histopathology, FA-treated OLETF rats showed decreased glomerular basement membrane thickness, glomerular volume, and mesangial matrix expansion. FA treatment decreased oxidative stress markers and MCP-1 levels in $24 \mathrm{~h}$ urine of rats and supernatants of cultured podocyte. In conclusion, it was suggested that FA have protective and therapeutic effects on diabetic nephropathy by reducing oxidative stress and inflammation.

Keywords: diabetes mellitus, experimental; diabetic nephropathies; ferulic acid; inflammation; oxidative stress

\section{Introduction}

Diabetic nephropathy is a major complication associated with type 2 diabetes and is a leading cause of end-stage renal disease (Kang et al., 2008). It is characterized functionally by proteinuria and albuminuria and pathologically by glomerular hypertrophy, mesangial expansion and tubulointerstitial fibrosis. These findings are closely related to the loss of renal function (Lee et al., 2007). The underlying mechanisms of the evolution of diabetic nephropathy are extremely complex, and several mediators have been implicated. Several growth factors or metabolic products, including transforming growth factor- $\beta 1$ (TGF- $\beta 1$ ), insulin-like growth factor-I, platelet-derived growth factor, angiotensin II, and advanced glycation end products, have been identified as contributing factors involved in the progression of diabetic glomerulopathy (Ziyadeh, 2004). Among these factors, reactive oxygen species are thought to play an important role in the development of diabetic nephropathy ( $\mathrm{Ha}$ and Lee, 2000). Hyperglycemia is the key initiating factor in the development of all chronic diabetic complications including diabetic nephropathy. It has been hypothesized that an increase in oxidative stress as a result of chronic hy- 
Table 1. Anthropometric and biochemical characteristics of experimental animals

\begin{tabular}{lccc}
\hline & CON & DM & DM + FA \\
\hline Initial body weight $(\mathrm{g})$ & $459.95 \pm 36.76$ & $597.06 \pm 33.66^{*}$ & $593.78 \pm 54.70^{*}$ \\
Final body weight $(\mathrm{g})$ & $540.30 \pm 47.76$ & $502.39 \pm 44.24$ & $627.67 \pm 91.46^{*, \dagger}$ \\
Left kidney Wt /BW $(100 \mathrm{~g})$ & $0.26 \pm 0.01$ & $0.46 \pm 0.04^{*}$ & $0.33 \pm 0.06^{*, \dagger}$ \\
Right kidney Wt/BW $(100 \mathrm{~g})$ & $0.22 \pm 0.01$ & $0.40 \pm 0.04^{*}$ & $0.29 \pm 0.05^{*, \dagger}$ \\
Insulin $(\mathrm{ng} / \mathrm{ml})$ & $2.56 \pm 1.25$ & $0.81 \pm 0.47^{\star}$ & $2.25 \pm 1.08^{\dagger}$ \\
KITT $(\% / \mathrm{min})$ & $4.20 \pm 0.58$ & $1.44 \pm 1.65^{*}$ & $4.64 \pm 0.66^{\dagger}$ \\
HOMA-BETA & $2946.87 \pm 1909.29$ & $74.93 \pm 50.01^{*}$ & $659.01 \pm 574.33^{\dagger}$ \\
HOMA-IR & $30.53 \pm 14.61$ & $34.71 \pm 24.82$ & $45.24 \pm 21.31$ \\
Adiponectin $(\mu \mathrm{g} / \mathrm{ml})$ & $7.59 \pm 0.26$ & $5.01 \pm 0.17^{*}$ & $6.31 \pm 0.25^{*, \dagger}$ \\
\hline
\end{tabular}

CON, control; DM, diabetes; FA, ferulic acid; BW, body weight. The values are mean \pm S.D. ${ }^{*} P<0.05$ compared with CON, ${ }^{\dagger} P<0.05$ compared with DM.

perglycemia activates several signaling pathways that alter gene expression (Chiu et al., 2009). Recent studies have suggested that inflammation plays a role in the progression of diabetic nephropathy (Fujita et al., 2008; Ko et al., 2008). Accordingly, many studies have focused on slowing down the progress of diabetic nephropathy by reducing oxidative stress as well as controlling blood glucose and blood pressure levels. Antioxidants suppress high glucose induced extracellular matrix protein synthesis in mesangial cells (Ha and Lee, 2000). In spite of the intensive control of blood glucose and blood pressure, diabetic nephropathy remains an important clinical problem. Therefore, new therapeutic drugs for controlling diabetic nephropathy are needed.

Ferulic acid (FA) is a phenolic acid found in the seeds and leaves of most plants. Rice bran in particular has many types of phenolic acids and concurrent biological activities. Moreover its chemical structure strongly resembles that of curcumin, the substance responsible for the yellow color of the spice turmeric. FA supplementation at relatively low doses increases the activities of antioxidant enzymes, thereby neutralizing free radicals which, in diabetics, are the primary cause of accelerated tissue damage (Srinivasan et al., 2007). Previous studies reported that FA is an antioxidant that neutralizes free radicals such as superoxide, nitric oxide and hydroxyl radicals that may cause oxidative damage to cell membranes and DNA (Kanski et al., 2002; Ha et al., 2008). FA provides meaningful synergistic protection against oxidative stress in the skin and should protect against photoaging and skin cancer (Lin et al., 2005), hypoglycemic and hypolipidemic effects (Sri Balasubashini et al., 2003; Ohnishi et al., 2004; Jung et al., 2007), hypotensive effects (Suzuki et al., 2002), and anti-inflammatory effects (Yagi and Ohishi, 1979).

The Otsuka Long-Evans Tokushima Fatty (OLETF) rat is an inbred strain that spontaneously develops type 2 diabetes and subsequently progresses to diabetic glomerulosclerosis. At 12 to 20 weeks of age, rats experience insulin resistance and mild obesity and after 18 weeks of age, rats show late-onset hyperglycemia with complications related to chronic diabetes that are similar to those of human type 2 diabetes. In this study, we investigated the therapeutic effects of FA on oxidative stress and inflammation by characterizing morphological changes and expression changes of TGF- $\beta 1$ and type IV collagen in the kidneys of type 2 diabetic OLETF rats.

\section{Results}

\section{Clinical characteristics of experimental animals}

The initial body weights of the diabetic control (DM) and FA-treated diabetic (DM + FA) group were significantly higher than those of the control Long-Evans Tokushima Otsuka (LETO) group. However, the weights of diabetic control group was significantly decreased at 45 weeks compared with FA-treated diabetic group, although the amount of diet was not different between diabetic control group and FA-treated diabetic group (62.00 \pm 4.14 $g$ vs $49.53 \pm 14.23 \mathrm{~g}, P=0.071)$. Weights of the kidney per body weight in FA-treated diabetic rats were markedly lower than in the diabetic control group (Table 1). Changes in plasma glucose during the intraperitoneal glucose tolerance test (IPGTT) in each experimental group at 45 weeks of age are illustrated in Figure 1A. Plasma glucose level was significantly decreased in the FA-treated diabetic group compared to the diabetic control group. Plasma glucose levels during the intravenous insulin tolerance test (IVITT) for each experimental group are shown in Figure 1B. Measured Kitt and homeostatic model assessment beta cell function 


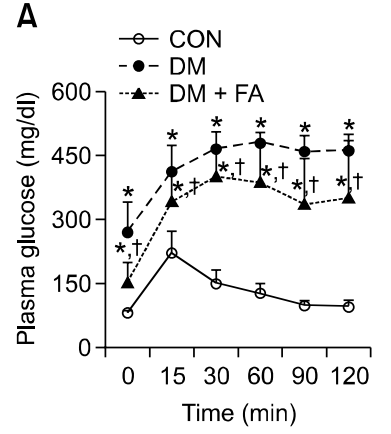

B

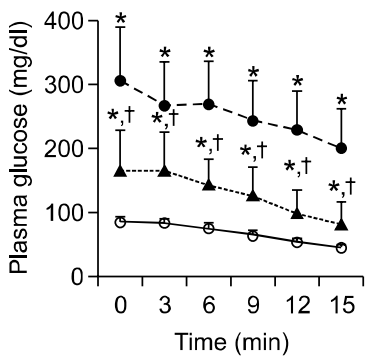

Figure 1. Blood glucose levels. (A) Using the intraperitoneal glucose tolerance test (IPGTT), the plasma glucose level of the FA-treated diabetic group was significantly decreased against the diabetic control group. (B) The plasma glucose level of the diabetic control group was significantly increased during IVITT periods compared to the control LETO group but the FA-treated diabetic group was decreased compared to the diabetic control group. CON, control group; DM, diabetic group; FA, ferulic acid treated diabetic group. Data express mean $\pm \mathrm{SD}$. ${ }^{*} P<0.05$ compared with $\mathrm{CON},{ }^{\dagger} P<0.05$ compared with DM.

(HOMA-beta) and homeostatic model assessment insulin resistance (HOMA-IR) values were shown in Table 1. The plasma glucose levels of the diabetic control group were significantly increased during IVITT periods compared to the control LETO group, but glucose levels in the FA-treated diabetic group was decreased compared to the diabetic control group. The Kitt and HOMA-beta values of the FA-treated diabetic group were significantly decreased compared to the diabetic control group. No differences were observed in the HOMA-IR values among these groups. Adiponectin serum level in the FA-treated diabetic group was higher than the diabetic control group (Table 1). At 25 weeks of age, $24 \mathrm{~h}$ urinary albumin excretion and albumin creatinine ratios (ACR) did not vary among the experimental rat groups. At 45 weeks of

Table 2. Changes of $24 \mathrm{~h}$ urinary albumin (mg/day) and ACR (mg/mgCr)

\begin{tabular}{lccc}
\hline & \multicolumn{3}{l}{24 h urinary albumin $(\mathrm{mg} /$ day $)$} \\
\cline { 2 - 4 } & 25 week & 37 week & 45 week \\
\hline CON & $1.05 \pm 0.57$ & $1.92 \pm 1.08$ & $0.85 \pm 0.40$ \\
DM & $12.35 \pm 6.97^{*}$ & $24.11 \pm 5.98^{*}$ & $26.76 \pm 9.46^{*}$ \\
DM + FA & $11.98 \pm 12.35^{*}$ & $21.14 \pm 14.15^{*}$ & $16.39 \pm 9.69^{*,}$ \\
\cline { 2 - 4 } & ACR $(\mathrm{mg} / \mathrm{mgCr})$ & \\
\cline { 2 - 4 } CON & $0.09 \pm 0.05$ & $0.13 \pm 0.07$ & $0.07 \pm 0.04$ \\
DM & $0.92 \pm 0.59^{*}$ & $1.79 \pm 0.66^{*}$ & $2.51 \pm 1.04^{*}$ \\
DM + FA & $0.88 \pm 0.67^{*}$ & $1.64 \pm 1.04^{*}$ & $1.49 \pm 0.90^{*,}$ \\
\hline
\end{tabular}

CON, control; DM, diabetes; FA, ferulic acid; $A C R$, albumin creatinine ratio. The values are mean \pm S.D. ${ }^{*} P<0.05$ compared with $\mathrm{CON},{ }^{\dagger} P<0.05$ compared with DM. age, $24 \mathrm{~h}$ urinary albumin excretion and ACRs of the FA-treated diabetic group were significantly lower than those of the diabetic control group (Table 2).

\section{Changes of $24 \mathrm{~h}$ urinary malondialdehyde (MDA) and monocyte chemoattractant protein-1 (MCP-1)}

At 45 weeks of age, $24 \mathrm{~h}$ urinary MDA increased in the diabetic control group compared to the control LETO group, but was effectively reduced by FA treatment (Figure 2A). Also, $24 \mathrm{~h}$ urinary MCP-1 expression decreased in the FA-treated diabetic group compared to the diabetic control group (Figure 2B).

\section{Glomerular basement membrane (GBM) thickening and podocyte morphometry for open slit pore numbers}

The GBM thickness was increased in the diabetic control group. However, in the diabetic control group treated with FA, diabetic GBM thickening was prevented (Figures $3 \mathrm{~A}$ and $3 \mathrm{~B}$ ). Podocyte foot process effacement was observed in the diabetic control group as expected. The number of slit pores between podocyte foot processes per unit length of GBM was significantly decreased in the diabetic control group, but these changes were significantly prevented by treatment with FA. (Figures $3 \mathrm{C}$ and $3 \mathrm{D})$.

\section{Glomerular volumes and glomerular mesangial index}

Using PAS staining of glomeruli, glomerular vol-
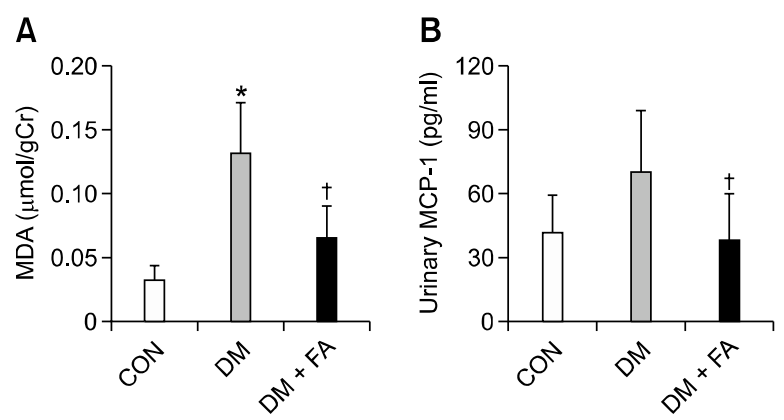

Figure 2. Changes of $24 \mathrm{~h}$ urinary malondialdehyde (MDA) and monocyte chemoattractant protein-1 (MCP-1) levels. (A) After the $24 \mathrm{~h}$ urine collected by metabolic cages, MDA was determined by HPLC. MDA was markedly increased in the diabetic control group compared to the control LETO group. However, MDA in the diabetic control group was significantly ameliorated by FA. (B) The MCP-1 measured using ELISA on $24 \mathrm{~h}$ urine samples, was markedly decreased in the FA-treated diabetic group compared to the diabetic control group. CON, control group; DM, diabetic group; FA, ferulic acid treated diabetic group; ${ }^{*} P<0.05$ compared with CON, ${ }^{\dagger} P<0.05$ compared with DM. 
A

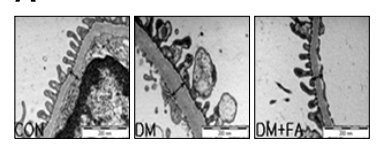

B

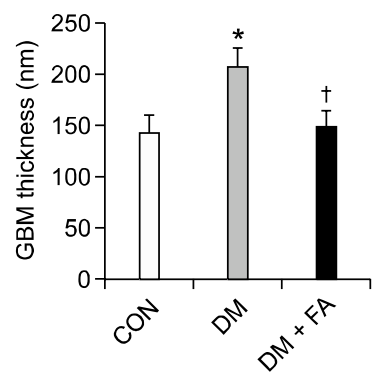

C

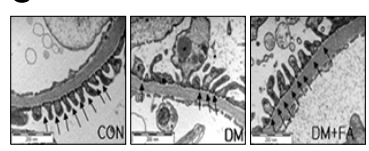

D

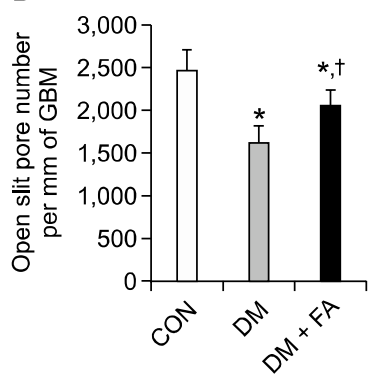

Figure 3. Glomerular basement membrane (GBM) thickening. (A) Representative electron photomicrographs showing the entire GBM, including the three layers (arrows). $\times 30,000$. (B) Diabetic control group developed GBM thickening versus control LETO group. Thickening was prevented in the diabetic rats that were treated with FA versus the diabetic control group. (C) Representative electron photomicrographsshowing open slit pores (arrows). $\times 30,000$. (D) As assessed by electron microscopy, the numbers of slit pores between podocyte foot processes were expressed per millimeter length of GBM to arrive at the mean numerical density for each of the three groups of rats. Diabetic control group had decreased open slit pore numbers, but these changes were significantly prevented by treatment with FA. CON, control group; DM, diabetic group; FA, ferulic acid treated diabetic group. ${ }^{*} P<0.05$ compared with $\mathrm{CON},{ }^{\dagger} P<0.05$ compared with DM.

umes were observed in the DM group compared to other groups. However, glomerular volumes decreased in the FA-treated diabetic group compared to the diabetic control group (Figures $4 \mathrm{~A}$ and $4 \mathrm{~B}$ ). Glomerular volumes in the diabetic control group showed expansion in the mesangial matrix compared to the control LETO group, which was ameliorated by FA treatments (Figure 4C).

\section{Immunohistochemical staining for TGF- $\beta 1$ and type IV collagen}

The immunohistochemical staining for TGF- $\beta 1$ and type IV collagen in the glomerular area of the diabetic control group showed darker brown pigmentation than for the other groups. Compared to the control LETO group, the optical density of immunohistochemical staining for renal TGF- $\beta 1$ and type IV collagen in the diabetic control group significantly increased and was restored in the FA-treated diabetic group (Figures 5 and 6).

\section{TGF- $\beta 1$ and type IV collagen protein expression}

Quantitative analysis revealed that expression of renal TGF- $\beta 1$ and type IV collagen was increased

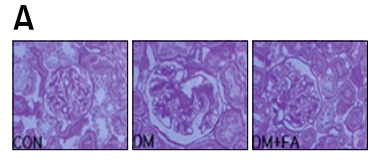

\section{B}

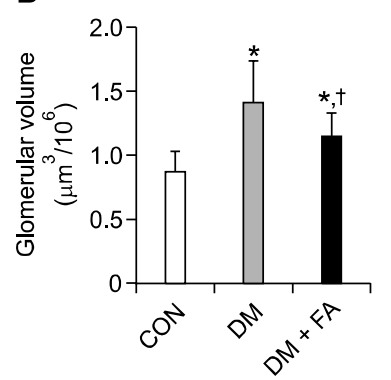

C

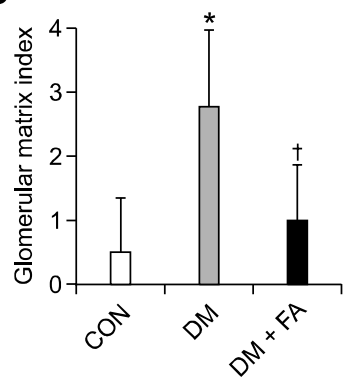

Figure 4. Glomerular volume and glomerular matrix index (GMI). (A) Representative examples of PAS staining of glomerulus. $\times 400$. (B) Glomerular volumes decreased in the FA-treated diabetic group compared to the diabetic control group. (C) The diabetic control group showed expansion in the mesangial matrix compared to the control LETO group, which was ameliorated by FA treatments. CON, control group; DM, diabetic group; FA, ferulic acid treated diabetic group. ${ }^{*} P<$ 0.05 compared with $\mathrm{CON},{ }^{\dagger} P<0.05$ compared with DM.
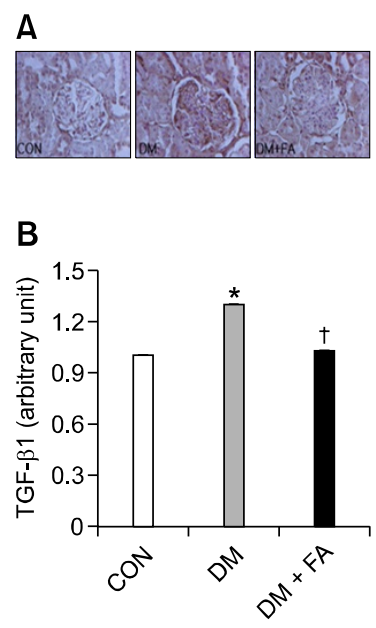

Figure 5. Immunohistochemical changes of glomerular transforming growth factor-beta1 (TGF- $\beta 1$ ). (A) Representative photomicrographs showing immunohistochemical staining of the glomerulus. $\times 400$. (B) Immunodetectable TGF- $\beta 1$, which was expressed in glomerular podocytes, was markedly decreased in the diabetic control group compared to the control LETO group. However, in the control group that was treated with FA, TGF- $\beta 1$ expression was effectively restored. CON, control group; DM, diabetic group; FA, ferulic acid treated diabetic group. ${ }^{*} P<$ 0.05 compared with $\mathrm{CON},{ }^{\dagger} P<0.05$ compared with DM.

in the diabetic control group, while it was decreased in the FA-treated diabetic group when compared to the control LETO group. However, between the diabetic control and FA-treated diabetic groups, there were no significant differences in the results (Figure 7). 
A

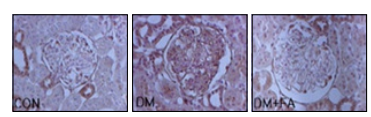

B

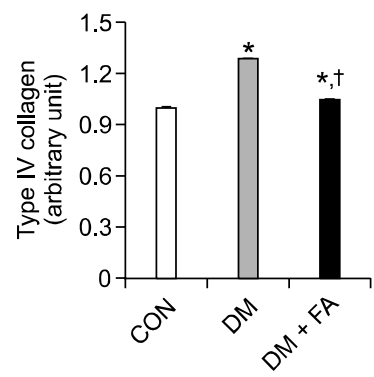

Figure 6. Immunohistochemical changes of glomerular type IV collagen. (A) Representative photomicrographs depicting immunohistochemical staining of the glomerulus. $\times 400$. (B) Immunodetectable type IV collagen, which was expressed in glomerular podocytes, was markedly decreased in the diabetic control group compared to the control LETO group. However, in the control group that was treated with FA, type IV collagen expression was effectively restored. CON, control group; DM, diabetic group; FA, ferulic acid treated diabetic group. ${ }^{*} P<0.05 \mathrm{com}-$ pared with $\mathrm{CON},{ }^{\dagger} P<0.05$ compared with DM.

\section{ROS production and MCP-1 expression in cultured podocytes}

The ROS production induced by high glucose condition was significantly decreased by FA treatment in cultured podocytes (Figure 8A). Also, the increased MCP-1 level was significantly suppressed by FA treatment (Figure 8B).

\section{Discussion}

Previous studies of FA treatment describe reduced blood glucose levels (Jung et al., 2007) and weakened lipid peroxide by increasing the antioxidant capacity (Sri Balasubashini et al., 2003). These effects may be a suppression of the ROS by FA treatments. These studies reported that FA treatment effectively decreased blood pressure levels (Suzuki et al., 2002).

We used OLETF rats to determine whether FA has beneficial effects in type 2 diabetic rats. The OLETF rat model is characterized by mild obesity and late-onset hyperglycemia with complications related to chronic diabetes that are similar to those of human type 2 diabetes. Therefore, we investigated how FA impacts the progression of diabetic nephropathy in OLETF rats.

FA treatments in the diabetic rats effectively improved blood glucose levels, increased insulin sensitivity and insulin secretion of beta cells.
A

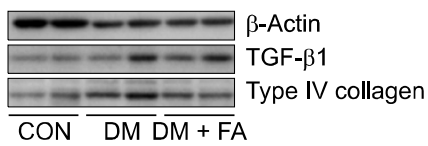

B

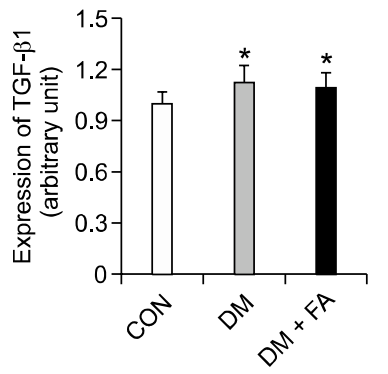

C

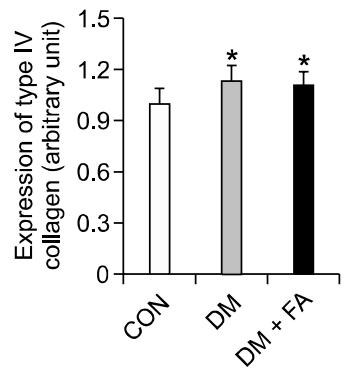

Figure 7. Western blot analysis for TGF- $\beta 1$ and type IV collagen. (A) Representative bands of western blot for TGF- $\beta 1$ and type IV collagen are shown. (B) The densitometric ratio of TGF- $\beta 1$ to $\beta$-actin was increased in the diabetic control group versus the control LETO group. TGF- $\beta 1$ protein was recovered by FA treatment in the diabetic control group. (C) The densitometric ratio of type IV collagen to $\beta$-actin was increased in the diabetic control group versus the control LETO group. Type IV collagen protein was restored by FA treatment in the diabetic control group. CON, control group; DM, diabetic group; FA, ferulic acid treated diabetic group. ${ }^{*} P<0.05$ compared with $\mathrm{CON}$.
A

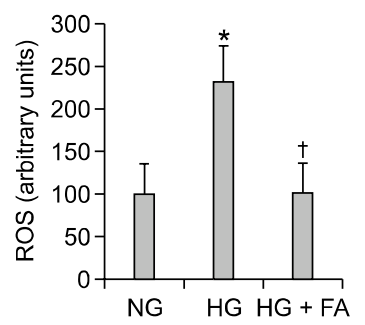

B

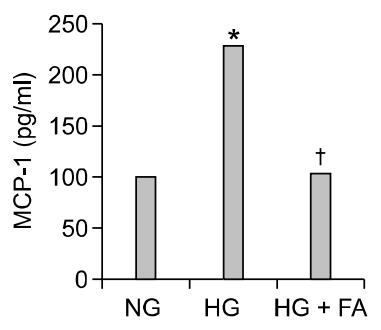

Figure 8. ROS production and MCP-1 expression in cultured podocytes. To induce ROS production, the podocytes were treated by high glucose media $(30 \mathrm{mM})$. The increased ROS production and MCP-1 levels were significantly suppressed by FA treatment $(10 \mu \mathrm{M})$. NG, normal glucose; $\mathrm{HG}$, high glucose; $\mathrm{HG}+\mathrm{FA}$, high glucose + ferulic acid. ${ }^{*} P<0.05 \mathrm{com}-$ pared with $\mathrm{CON},{ }^{\top} P<0.05$ compared with DM.

Adiponectin (Ohara et al., 2009) which is known to play an important role in the modulation of glucose metabolism, is increased by FA treatments. The FA-treated diabetic group showed no losses of body weight as the hyperglycemia improved and decreases of the urinary albumin and albumincreatinine ratio.

Oxidative stress is reported to play a key role in the progression of diabetic nephropathy (Evans et al., 2002) and induces MCP-1 expression in many types of cells (Chander et al., 2004). MCP-1 plays a crucial role in mesangial cell proliferation, glomerulosclerosis and renal fibrogenesis (Tanifuji et al., 2005). In our in vivo data, urinary MDA and 
MCP-1 levels were statistically decreased in the FA-treated diabetic group compared to the diabetic control group. Also, high glucose induced ROS production and MCP-1 expression was significantly suppressed by FA in cultured podocyte. These results suggest that $F A$ has strong antioxidant and anti-inflammatory effects.

Glomerular podocyte is known to play a critical role in kidney structure and urinary filtration. Podocyte foot process surrounds the outside of GBM formed slit diaphragm with adjacent podocyte foot process. This structure is very effective for the maximization of glomerular filtration. When kidney injury occurs, there are molecular composition changes of the slit diaphragm, podocyte foot process rearrangement and slit diaphragm fusion (Lee et al., 2003; Lee and Chung, 2007; Stitt-Cavanagh et al., 2009). In addition hyperglycemia, advanced glycation end product (AGE), ROS, and TGF- $\beta 1$ in diabetes mellitus could be important factors in the process of glomerular podocyte damage (Lee and Chung, 2007). We observed that GBM thickening was prevented and that the number of slit pores between podocyte foot processes per unit length of GBM was increased with FA treatment. Moreover, FA also suppressed glomerular hypertrophy and mesangial matrix expansion in the OLETF rat model.

Finally, TGF- $\beta 1$ has been reported to stimulate MCP-1 expression in mesangial cells (Cheng et al., 2005), to stimulate MCP-1 to promote collagen deposition and is known to be a final mediator of extracellular matrix expansion, collagen deposition and fibrogenesis in diabetic nephropathy (Schneider et al., 1999). To confirm these results, we measured the expression of TGF- $\beta 1$ and type IV collagen in the renal cortex and found that FA treatment reduced the expression of TGF- $\beta 1$ and type IV collagen.

Taken together, these results demonstrate that the renal protective effects of FA occur through improved glycemic control and renal structural changes, which are involved in the inhibition of oxidative stress, inflammation and the expression of TGF- $\beta 1$ and type IV collagen. Collectively, we could conclude that FA attenuates the development of diabetic nephropathy in type 2 diabetic OLETF rats and FA may be a potential new therapeutic drug for diabetic nephropathy in type 2 diabetic patients.

\section{Methods}

\section{Animal experiments}

All animal procedures were approved by the Institutional
Animal Care and Use Committee, Yonsei University, Wonju Campus. At 25 weeks, 18 male OLETF (Otsuka Pharmaceutical, Tokushima, Japan) and 9 male LETO rats were divided into three groups: diabetic control group, FA-treated diabetic group, and normal control group. Animals were housed at constant temperature $\left(20-22^{\circ} \mathrm{C}\right)$ and humidity $(50-60 \%)$ with a 12-h light and 12-h dark cycle. They were allowed free access to water and standard rat chow until 45 weeks of age. The experimental group received FA (10 mg/kg/day) by oral gavage tube from 26 weeks to 45 weeks. At the same time, the diabetic control and FA-treated diabetic group induced diabetes by $30 \%$ sucrose solution freely feeding. At 25,37 and 45 weeks, body weight, IPGTT and IVITT were measured and a 24-h urine sample was collected for assessment of albumin and creatinine (Exocell Nephrat; Exocell Inc., Philadelphia, PA) and MCP-1 (Invitrogen, Carlsbad, CA). Plasma insulin levels and plasma adiponectin levels were measured using ELISA kits (Shibayagi Co., Shibukawa, Japan, and AdipoGen Inc., Seoul, Korea).

\section{Kidney extraction}

At 45 weeks of age, experimental rats were anesthetized with Zoletil ${ }^{\mathbb{R}}$ (Virbac Laboratories, Carros, France) by intraperitoneal injection and both kidneys were extracted. One kidney was preserved using a quick freeze method with liquid nitrogen while the contralateral kidney was fixed with $4 \%$ paraformaldehyde for $48 \mathrm{~h}$, and then embedded in paraffin for histological examination and immunohistochemical staining of TGF- $\beta 1$ and type IV collagen.

\section{$24 \mathrm{~h}$ MDA assay}

A rapid and sensitive fluorometric HPLC method was used for the measurement of urine MDA. The mobile phase consisted of $5 \mathrm{mM}$ boric acid and $35 \%$ acetonitrile, pumped at a rate of $1.0 \mathrm{ml} / \mathrm{min}$ on a Water-Alliance 2696 system Symmetry $\mathrm{C}_{18}(5 \mathrm{~mm}$ particle size, $4.6 \mathrm{~nm} \times 150 \mathrm{~nm})$ with the column warmer set to $40^{\circ} \mathrm{C}$. Urine samples were treated with 2,4-dinitrophenylhydrazine and heat derivatized at $40^{\circ} \mathrm{C}$ for $40 \mathrm{~min}$ with $1 \mathrm{M} \mathrm{HCl} 50 \mathrm{ml}$ at an acid $\mathrm{pH}$. Samples were extracted with $\mathrm{n}$-butanol and $10 \mathrm{ml}$ of the extract was injected at 1-min intervals using an autosampler. An ultraviolet-visible spectrophotometer detector (Waters Inc., Milford, MA) was set at an excitation wavelength of $515 \mathrm{~nm}$ and emission of $553 \mathrm{~nm}$. Although the retention time was $1.87 \mathrm{~min}$, the absence of interfering peaks allowed analysis to be carried out in increments of 1 $\mathrm{min} / \mathrm{sample}$. Within-day variability in estimation was between 8.6 and $10.3 \%$. Between-day variability was $3.6-7.9 \%$. Recovery was between 88 and $101 \%$.

\section{Measurement of glomerular basement membrane (GBM) thickness}

Each specimen was thin-sectioned and examined under a JEOL transmission electron microscope (JEM-1200EX II, JEOL Ltd., Tokyo, Japan). Electron micrographs of five to ten glomeruli per kidney were randomly taken at $30,000 \times$ for each rat. Photomicrographs of the GBM were also ana- 
lyzed for the density of slit pores between the podocyte foot processes. The numbers of slit pore were counted and divided by the GBM length $(\mathrm{mm})$ to arrive at the linear density using an image analysis system $(\mathrm{GmbH}$, SIS, Minster, Germany).

\section{Measurement of glomerular volumes and glomerular matrix index}

Paraffin embedded tissues were cut into $5 \mu \mathrm{m}$ thick sections and stained with PAS stain. We examined these sections with an optical microscope that was equipped with a charge coupled device camera (Pulnix, Sunnyvale, CA) in order to obtain pictures of glomeruli, which were subsequently sent to a computer monitor. We measured 40 glomerular areas per rat using an image analysis system $(\mathrm{GmbH}, \mathrm{SIS})$. In addition, we calculated glomerular volume using the Weibel and Gomez formula (Lane et al., 1992): Glomerular volume $(\mathrm{Gv})=$ Area $^{1.5} \times 1.38 / 1.01$ (1.38: shape coefficient, 1.01: size distribution coefficient).

The glomerular matrix index (GMI) measured mesangial matrix collected for the glomerular area (Sassy-Prigent et al., 1995; Naito et al., 2004). The degree of mesangial matrix expansion was assessed using a semiquantitative scoring method (Nam et al., 2009; Sung et al., 2010): grade 0 , normal glomeruli; grade 1 , mesangial expansion area up to $25 \%$ (minimal); grade 2, 25-50\% (moderate); grade 3, 50-75\% (moderate-severe); grade 4, 75-100\% (severe). The glomerular mesangial expansion index (GMI) was calculated as the average of 40 glomeruli on each kidney slide.

\section{Immunohistochemical staining for TGF- $\beta 1$ and type IV collagen}

Kidneys were fixed in $4 \%$ paraformaldehyde and embedded in paraffin. Each slide was prepared with $5-\mu \mathrm{m}$ thick-sections and placed on slides and the paraffin was removed in order to carry out immnunohistochemical staining. They were then dehydrated in xylene and graded alcohols, and were immersed in distilled water. Kidney sections were transferred to $10 \mathrm{~mm} / \mathrm{L}$ of $\mathrm{pH} 6.0$ citrated buffer solution for $10 \mathrm{~min}$. After being washed with water, $0.05 \%$ of $\mathrm{H}_{2} \mathrm{O}_{2}$-methanol was applied for $20 \mathrm{~min}$. We used transforming growth factor-beta1 (TGF- $\beta 1$ ) and type IV collagen antibodies (Santa Cruz Biotechnology Inc., Santa Cruz, CA) diluted 1:500 as the primary antibodies. A rat biotinylated secondary antibody was added (Santa Cruz Biotechnology Inc.) and antibody binding was visualized using the avidin and biotinylated horseradish peroxidase reaction. To evaluate the TGF- $\beta 1$ and type IV collagen staining, we observed stained slides using a light-microscope adhered to a charge-coupled camera device (Pulnix, Sunnyvale, CA). We measured the optical density of stained TGF- $\beta 1$ and type IV collagen using an image analysis system (GmbH, SIS).

\section{Protein expression for TGF- $\beta 1$ and type IV collagen}

The kidney cortex was homogenized by the TissueLyser II (QIAGEN GmbH, Haan, Germany) in a RIPA lysis buffer (Thermo Scientific, Rockford, IL). Protein concentration was measured by the Bio-Rad protein assay kit (Bio-Rad Laboratories Inc., Hercules, CA). Thirty micrograms of each protein sample were run on a $10 \%$ SDS-polyacrylamide gel under denaturing conditions. The proteins were transferred onto a polyvinylidene difluoride membrane (Immobilon; Millipore, Bedford, MA) for $90 \mathrm{~min}$ at $280 \mathrm{~mA}$. After the filter was blocked by incubating the membranes with $5 \%$ skim milk for $1 \mathrm{~h}$ at room temperature, the membrane was hybridized with a rabbit anti- $\beta$-actin antibody (Cell Signaling Technology Inc., Denvers, MA) or rabbit anti-TGF- $\beta 1$ and rabbit anti-type IV collagen antibodies (Santa Cruz Biotechnology Inc.) in a blocking buffer overnight at $4^{\circ} \mathrm{C}$. The filter was then incubated with a horseradish peroxidase-conjugated secondary antibody for $60 \mathrm{~min}$ at room temperature. Specific signals were detected using the ECL method (Santa Cruz Biotechnology).

\section{ROS production and MCP-1 expression in cultured podocytes}

Conditionally immortalized mouse podocytes (gift of Dr. Mundel P, University of Miami, Miami, FL) were cultured as previously described (Lee et al., 2006, 2009). The cells were allowed to differentiate at $37.5^{\circ} \mathrm{C}$ without $\gamma$-interferon in DMEM containing $5.5 \mathrm{mM}$ glucose. Differentiated podocytes were synchronized into quiescence by growing cells in serum-free medium for $24 \mathrm{~h}$. The cultured podocyte was treated by high glucose media $(30 \mathrm{mM})$ with or without FA $(10 \mu \mathrm{M})$. After $24 \mathrm{~h}$, we measured MCP-1 levels in supernatant by ELISA kit (R\&D, Minneapolis, MN). For evaluation of ROS production, cultured podocytes were washed by PBS twice and added dichlorofluorescein diacetate (DCF-DA) $10 \mu \mathrm{M}$ at $37.5^{\circ} \mathrm{C}$ condition for $30 \mathrm{~min}$. We quantitatively analyzed the flurocence by flurometer.

\section{Statistic analysis}

All results are presented as means $\pm S D$. Data was analyzed using SPSS version 17.0. Statistical significance was evaluated using analysis of variance with Tukey's post hoc test (multiple comparisons). $P$ values of less than 0.05 were considered statistically significant.

\section{References}

Chander PN, Gealekman O, Brodsky SV, Elitok S, Tojo A, Crabtree M, Gross SS, et al. Nephropathy in zucker diabetic fat rat is associated with oxidative and nitrosative stress: prevention by chronic therapy with a peroxynitrite scavenger ebselen. J Am Soc Nephrol 2004;15:2391-403

Cheng J, Diaz Encarnacion MM, Warner GM, Gray CE, Nath KA, Grande JP. TGF-beta1 stimulates monocyte chemoattractant protein-1 expression in mesangial cells through a phosphodiesterase isoenzyme 4-dependent process. Am J Physiol Cell Physiol 2005;289:C959-70

Chiu J, Khan ZA, Farhangkhoee H, Chakrabarti S. Curcumin prevents diabetes-associated abnormalities in the kidneys by inhibiting p300 and nuclear factor- $\kappa$ B. Nutrition 2009; 


\section{$25 \cdot 964-72$}

Evans JL, Goldfine ID, Maddux BA, Grodsky GM. Oxidative stress and stress-activated signaling pathways: a unifying hypothesis of type 2 diabetes. Endocr Rev 2002;23:599-622

Fujita A, Sasaki H, Doi A, Okamoto K, Matsuno S, Furuta H, Nishi M, Nakao T, Tsuno T, Taniguchi H. Ferulic acid prevents pathological and functional abnormalities of the kidney in Otsuka Long-Evans Tokushima Fatty diabetic rats. Diabetes Res Clin Pract 2008;79:11-7

$\mathrm{Ha} \mathrm{H}$, Hwang I, Park J, Lee H. Role of reactive oxygen species in the pathogenesis of diabetic nephropathy. Diabetes Res Clin Pract 2008;82:S42-5

$\mathrm{Ha} \mathrm{H}$, Lee HB. Reactive oxygen species as glucose signaling molecules in mesangial cells cultured under high glucose. Kidney Int Suppl 2000;77:S19-25

Jung EH, Kim SR, Hwang IK, Ha TY. Hypoglycemic effects of a phenolic acid fraction of rice bran and ferulic acid in c57bl/ksj-db/dbmice. J Agric Food Chem 2007;55:9800-4

Kang ES, Lee GT, Kim BS, Kim CH, Seo GH, Han SJ, Hur $\mathrm{KY}$, Ahn CW, Ha H, Jung M, Ahn YS, Cha BS, Lee HC. Lithospermic acid $B$ ameliorates the development of diabetic nephropathy in OLETF rats. Eur J Pharmacol 2008;579: 418-25

Kanski J, Aksenova M, Stoyanova A, Butterfield DA. Ferulic acid antioxidant protection against hydroxyl and peroxyl radical oxidation in synaptosomal and neuronal cell culture systems in vitro: structure-activity studies. J Nutr Biochem 2002;13:273-81

Ko GJ, Kang YS, Han SY, Lee MH, Song HK, Han KH, Kim HK, Han JY, Cha DR. Pioglitazone attenuates diabetic nephropathy through an anti-inflammatory mechanism in type 2 diabetic rats. Nephrol Dial Transplant 2008;23: 2750-60

Lane PH, Steffes MW, Mauer SM. Estimation of glomerular volume: a comparison of four methods. Kidney Int 1992; 41:1085-9

Lee EY, Song JS, Chung $\mathrm{CH}$, Hong SY. Mechanism of podocyte injury in diabetic nephropathy. Korean Diabetes $\mathrm{J}$ 2003;27:343-51

Lee EY, Chung CH, Kim JH, Joung HJ, Hong SY. Antioxidants ameliorate the expression of vascular endothelial growth factor mediated by protein kinase $\mathrm{C}$ in diabetic podocytes. Nephrol Dial Transplant 2006;21:1496-503

Lee EY, Lee MY, Hong WY, Chung $\mathrm{CH}$, Hong SY. Blockade of oxidative stress by vitamin c ameliorates albuminuria and renal sclerosis in experimental diabetic rats. Yonsei Med J 2007;48:847-55

Lee $\mathrm{EY}$, Chung $\mathrm{CH}$. The role of glomerular podocytes in diabetic nephropathy. Korean Diabetes J 2007;31:451-4

Lee EY, Chung $\mathrm{CH}$, Khoury CC, Yeo TK, Pyagay PE, Wang $A$, Chen $S$. The monocyte chemoattractant protein-1/CCR2 loop, inducible by TGF- $\beta$, increases podocyte motility and albumin permeability. Am J Physiol Renal Physiol 2009;297:F85-94

Lin FH, Lin JY, Gupta RD, Tournas JA, Burch JA, Selim MA, Monteiro-Riviere NA, Grichnik JM, Zielinski J, Pinnell SR.
Ferulic acid stabilizes a solution of vitamins $C$ and $E$ and doubles its photoprotection of skin. J Invest Dermatol 2005;125:826-32

Naito Y, Uchiyama K, Aoi W, Hasegawa G, Nakamura N, Yoshida N, Maoka T, Takahashi J, Yoshikawa T. Prevention of diabetic nephropathy by treatment with astaxanthin in diabetic $\mathrm{db} / \mathrm{db}$ mice. Biofactors 2004;20:49-59

Nam SM, Lee MY, Koh JH, Park JH, Shin JY, Shin YG, Koh $\mathrm{SB}$, Lee EY, Chung $\mathrm{CH}$. Effects of NADPH oxidase inhibitor on diabetic nephropathy in OLETF rats: The role of reducing oxidative stress in its protective property. Diabetes Res Clin Pract 2009;83:176-82

Ohara K, Uchida A, Nagasaka R, Ushio H, Ohshima T. The effects of hydroxycinnamic acid derivatives on adiponectin secretion. Phytomedicine 2009;16:130-7

Ohnishi M, Matuo T, Tsuno T, Hosoda A, Nomura E, Taniguchi $H$, Sasaki $H$, Morishita $H$. Antioxidant activity and hypoglycemic effect of ferulic acid in STZ-induced diabetic mice and KK-Ay mice. Biofactors 2004;21:315-9

Sassy-Prigent $C$, Heudes $D$, Jouquey $S$, Auberval D, Belair MF, Michel O, Hamon G, Bariety J, Bruneval P. Morphometric detection of incipient glomerular lesions in diabetic nephropathy in rats. Protective effects of ACE inhibition. Lab Invest 1995;73:64-71

Schneider A, Panzer U, Zahner G, Wenzel U, Wolf G, Thaiss F, Helmchen U, Stahl RA. Monocyte chemoattractant protein-1 mediates collagen deposition in experimental glomerulonephritis by transforming growth factor-beta. Kidney Int 1999;56:135-44

Sri Balasubashini M, Rukkumani R, Menon VP. Protective effects of ferulic acid on hyperlipidemic diabetic rats. Acta Diabetol 2003;40:118-22

Srinivasan M, Sudheer AR, Menon VP. Ferulic acid: therapeutic potential through its antioxidant property. J Clin Biochem Nutr 2007;40:92-100

Stitt-Cavanagh E, MacLeod L, Kennedy C. The podocyte in diabetic kidney disease. ScientificWorldJournal 2009;9: 1127-39

Sung JK, Koh JH, Lee MY, Kim BH, Nam SM, Kim JH, Yoo $\mathrm{JH}$, Kim SH, Hong SW, Lee EY, Choi R, Chung CH. Aldose reductase inhibitor ameliorates renal vascular endothelial growth factor expression in streptozotocin-induced diabetic rats. Yonsei Med J 2010;51:385-91

Suzuki A, Kagawa D, Fujii A, Ochiai R, Tokimitsu I, Saito I. Short- and long-term effects of ferulic acid on blood pressure in spontaneously hypertensive rats. Am J Hypertens 2002; 15:351-7

Tanifuji C, Suzuki Y, Geot WM, Horikoshi S, Sugaya T, Ruiz-Ortega M, Egido J, Tomino Y. Reactive oxygen species-mediated signaling pathways in angiotensin II-induced MCP-1 expression of proximal tubular cells. Antioxid Redox Signal 2005;7:1261-8

Yagi K, Ohishi N. Action of ferulic acid and its derivatives as antioxidants. J Nutr Sci Vitaminol (Tokyo) 1979;25:127-30

Ziyadeh FN. Mediators of diabetic renal disease: the case for tgf-Beta as the major mediator. J Am Soc Nephrol 2004;15: S55-7 